\title{
Assessment of self care patients' knowledge regarding chronic Lower Limb Ischemia at Assiut University Hospital.
}

\author{
Amna Abdulla Desoky, Magda Abd- Elaziz Mohamed, Mostafa Saad Khhalil \& Amal Mohammko.ed \\ Ahmed. \\ Assistant lecturer of Medical Surgical Nursing, Faculty of Nursing, Assiut University.. \\ Professor of Medical Surgical Nursing Department, Faculty of Nursing, Ain Shams University. \\ Professor of Vascular Surgery Department, Faculty of Medicine, Assiut University. \\ Assistant Professor of Medical Surgical Nursing Department, Faculty of Nursing, Aswan University.
}

\begin{abstract}
Chronic lower limb ischemia is a prevalent systemic atherosclerotic disease that impairs a patient's health condition and untreated disease can lead to limb loss. The aim: of the study was to assess patients' knowledge about self care regarding chronic lower limb ischemia at Assuit University Hospital. Descriptive research design: was utilized in the study. The study was conducted at vascular surgery department of Assuit University Hospital.

A convenience: sample: of sixty adult male and female patients diagnosed with chronic lower limb ischemia. The following tools were utilized for data collection; first tool: "Patients' structured assessment", second tool: "self care patients' knowledge regarding chronic lower limb ischemia questionnaires ". Data was collected \& analyzed.

Results: showed that; all patients had unsatisfactory level of knowledge about self care regarding their condition. In conclusion; teaching theses patients is necessary in oreder to be equipped with sufficient knowledge to manage this chronic disorder.
\end{abstract}

\section{Key words: Self Care Patients\& Knowledge Chronic Lower Limb Ischemia.}

\section{Introduction}

Peripheral vascular disease is commonly used to refer to peripheral arterial disease (PAD) outside the heart and brain. Arterial disorders represent the most common cause of morbidity and death in western societies. The disease prevalence increases with age and $12-20 \%$ of Americans age 65 and older (4.5 to 7.6 million) have PAD. As the population ages, the prevalence could reach 9.6 to 16 million in those age 65. Chronic lower limb ischemia known as peripheral arterial disease. (Rutherford, 2010).

Ischemia is a condition in which there is inadequate blood flow and oxygen to a specific part of the body. It can occur in any muscle group, organ, or tissue in the body. For example, in the lower extremities, ischemia can cause claudication in peripheral arterial disease (PAD) or may cause critical limb ischemia (CLI) in severe cases. In the heart, ischemia can cause angina or a heart attack; in the brain, ischemia may cause stroke or transient ischemic attacks and; in the intestines it is known as mesenteric ischemia and can cause severe abdominal pain. (Gey \& Manngold, 2008)

The manifestations of chronic lower limb ischemia usually include some type of pain; they range from no symptoms to intermittent claudication to critical limb ischemia. The common major manifestations of CLI are; rest pain, ischemic ulceration, or gangrene of the forefoot or toes. Walking is a fundamental human requirement. Ischemia is the most common cause of loss of normal walking ability seen by the vascular specialist. In addition to affecting the legs, it is associated with high systemic morbidity and mortality. (Hirsch, 2009)

Diagnosis of lower limb ischemia is typically based on the present history, physical findings, and diagnostic procedures such as ankle brachial index, doppler ultrasound, angiography, computed tomography, and magnetic resonance angiography. Treatment of lower limb ischemia is: risk factors modification, pharmacological therapies, endovascular intervention such as Percutanous transluminal angioplasty (PTA) and surgery. The overall goals of treatment are to reduce symptoms, improve quality of life, and prevent complications. (Linsky, 2010)

The nurse can improve the patients' self care by teaching them about methods of controlling pain associated with peripheral arterial disease, giving them health teaching about risk factors modification, exercise programs that improve peripheral arterial circulation, and provides care to the patients who will undergo surgical or interventional procedures. Also the nurse ensures that the patient has the knowledge and the ability to assess for potential complications. (Pudner, 2010)

This study aimed to assess self care patients' knowledge regarding chronic lower limb ischemia at Assuit University Hospital. 


\section{Research question}

What is the self care patients' knowledge regarding chronic lower limb ischemia?

\section{Patients and method}

\section{Research design}

Descriptive research design was utilized in this study.

\section{Setting}

The study was conducted at the vascular surgery department of Assuit University Hospital.

\section{Sample}

A convenience sample of sixty adult male and female patients diagnosed with chronic lower limb ischemia with the following inclusion criteria: Moderate to severe claudication and/or critical limb ischemia, treated conservatively or with Percutanous transluminal angioplasty (PTA), and agreed to participate in the study. And excluded for the following: Sever cardiac (heart failure, recent ischemic attack and myocardial infarction), Cerebro vascular abnormalities (transient ischemic attack, cerebro vascular accident), Previous endovascular procedures as percutaneous tranluminal angioplasty or vascular surgery.

Tools for data collection

Tool I: "Patients' structured assessment"

This tool was developed by researcher to assess socio demographic of pahent characteristic and clinical biomedical data structural assessment, it consists of two parts:

\section{Part one}

It was developed to assess the patients' sociodemographic characteristics as name, age, sex, marital status, occupation and education.

\section{Part two (medical data):-}

It includes structured items to identify the patients' needs:-

1. Past, present, and family history, past history such as previous exposure to either any cardiac disorders as angina, myocardial infarction, dysarrythmmia, and heart failure. Or cerebral disorders as transient ischemic attack and cerebrovascular accident. Present history; as presence of any risk factors as smoking, hypertension and diabetes.

2. Physical examination: Inspection, palpation, and auscultation were used to examine the lower extremities. The lower extremities were inspected for presence of trophic changes, ulceration, and gangrene. Palpation was done to detect presence or absence of pulse, if the pulse was undetected by palpation, the pulse was assessed by doppler. Auscultation was done for detection presence or absence of bruit.
3. Assessment of expected complications that will develop after interventional (PTA) therapy such as hematoma, arterial thrombosis and occlusion. (Annex I).

Tool II: Interview questionnaires about: Self care patients' knowledge regarding chronic lower limb ischemia".

It includes open and closed end questions to assess patients' knowledge about self care regarding chronic lower limb ischemia with total questions were 38 as the following:

1. Patients' knowledge about anatomy and physiology of peripheral arterial system. It consists of four (4) questions.

2. Patients' knowledge about disease "chronic lower limb ischemia" it consists of ten (10) questions.

3. Patients' knowledge about methods of improving circulation in the lower limb. It consists of eight (8) questions.

4. Patients' knowledge about prevention of weakness of circulation in the lower limb. It consists of eight (8) questions.

5. Patients' knowledge about foot care. It consists of five (5) questions.

6. Patients' knowledge about diet, it consists of three (3) questions.

\section{Scoring of this tool (tool II)}

Each right answer of open questions was given one mark. The total number of marks was (38) mark. This system translated in results into satisfactory and unsatisfactory, Patients who obtained less than $60 \%$ (22.8) were considered having unsatisfactory level of knowledge. While those who obtained more than 60 $\%$ (22.8) were considered having satisfactory level of knowledge. (Annex II).

\section{Operational design}

It includes preparatory phase, field work phase" implementation phase" and evaluation phase.

\section{Preparatory phase}

After reviewing the recent related literatures; study tools were developed.

\section{Content validity}

Content validity was done by five expertise (two nursing staff) from the medical surgical nursing field and (three vascular surgeons specialists) from the medical field to test relevance of the contents, clarity and comprehensiveness of the tools.

\section{Pilot study}

Pilot study was conducted on $10 \%$ (6 patients) of sample to evaluate the reliability, feasibility applicability and clarity of the tools, estimate the time needed for data collection, and test the feasibility of 
conducting the research after analyzing the pilot study results.

\section{Field work "implementation phase}

An official letter was issued from the Dean of the faculty of nursing to head of Assuit university hospitals and the head of the vascular surgery department. The researcher met vascular surgeons' specialists for explaining the purpose of the study to gain their cooperation.

\section{Ethical considerations}

At initial interview, each patient was informed with the purpose of the study. And the researcher emphasized that the participant is voluntary and confidentiality and anonymity of the subjects knowledge will be assured through coding of all data. All patients were interviewed by researcher during admission period to assess their self care knowledge regarding chronic lower limb ischemia by using the above mentioned tools.

\section{Statistical design}

Data was collected and analyzed using the following tests frequency, percentage, mean, and standard deviation. A probability level of 0.05 was adopted as a level of significance for testing the research hypothesis.

\section{Results}

Table (1): Distribution of studied sample as regarding to their sociodemographic characteristics.

\begin{tabular}{|l|c|c|c|}
\hline \multirow{2}{*}{ Characteristics } & \multicolumn{2}{|c|}{ Patients n= (60) } & \multirow{2}{*}{ Mean \pm SD } \\
\cline { 2 - 3 } Age (years) & No & $\%$ & \\
< 40 & 4 & 6.66 & \multirow{2}{*}{$59.73 \pm 11.17$} \\
$40-50$ & 7 & 41.66 & \\
$50-60$ & 25 & 40.00 & \\
60 \& above & 24 & & \\
\hline Sex & & 65.00 & \\
Male & 39 & 35.00 & \\
Female & 21 & & \\
\hline Residence & & 81.7 & \\
Rural & 49 & 18.3 & \\
Urban & 11 & 3.33 & \\
\hline Marital status & 2 & 71.66 & \\
Single & 43 & - & \\
Married & - & 25.00 & \\
Divorced & 15 & 61.66 & \\
Widow/widew & & 11.66 & \\
\hline Educational level & 37 & 6.66 & \\
Illiterate & 7 & 18.3 & \\
Read and write & 4 & 1.66 & \\
Preparatory & 11 & 18.3 & \\
Secondary & 1 & 6.66 & \\
University & 11 & 31.66 & \\
\hline Occupation & 4 & 11.66 & \\
Office work & 19 & & \\
Machinery work & 7 & & \\
Farmer & & & \\
House wife & & & \\
Not work & & & \\
\hline
\end{tabular}


Part II: Medical data.

Table (2): Distribution of studied sample as regarding to associated systemic disorders.

\begin{tabular}{|l|c|c|}
\hline \multirow{2}{*}{ Systemic disorders } & \multicolumn{2}{|c|}{ Patients n= (60) } \\
\cline { 2 - 3 } & No & $\%$ \\
\hline Cardiac disorders & & 31.66 \\
Yes & 19 & 68.33 \\
No & 41 & 23.33 \\
\hline Neurologic disorders & 14 & 76.66 \\
Yes & 46 & \\
No & & \\
\hline
\end{tabular}

Table (3): Distribution of studied sample as regarding to their risk factors.

\begin{tabular}{|l|c|c|}
\hline \multirow{2}{*}{ Risk factors } & \multicolumn{2}{|c|}{ Patients n= (60) } \\
\cline { 2 - 3 } & No & $\%$ \\
\hline Family history & & \\
Yes & 25 & 41.66 \\
No & 35 & 58.33 \\
\hline Smoking & 28 & 46.66 \\
Yes & 32 & 53.33 \\
No & & \\
\hline Hypertension & 29 & 48.33 \\
Yes & 31 & 51.66 \\
No & 2 & 3.33 \\
\hline Hypertension & 27 & 45.00 \\
Controlled & & \\
Not controlled & 39 & 65.00 \\
\hline Diabetes Mellitus & 21 & 35.00 \\
Yes & & \\
No & 5 & 8.33 \\
\hline Diabetes Mellitus & 34 & 56.66 \\
Controlled & & \\
Not controlled & & \\
\hline
\end{tabular}

Table (4): Distribution of studied sample as regarding to signs and symptoms of chronic lower limb ischemia.

\begin{tabular}{|l|c|c|}
\hline \multirow{2}{*}{ Signs and symptoms. } & \multicolumn{2}{|c|}{ Patients n= (60) } \\
\cline { 2 - 3 } & No & No \\
\hline Intermittent claudication & 5 & 8.33 \\
Present & 55 & 91.66 \\
Absent & & \\
\hline Rest pain & 51 & 85.00 \\
Present & 9 & 15.00 \\
Absent & 24 & 40.00 \\
\hline Ulceration & 36 & 60.00 \\
Present & & \\
Absent & 20 & 33.33 \\
\hline Gangrene & 23 & 38.33 \\
No & 17 & 28.33 \\
Dry & & \\
Wet & & \\
\hline
\end{tabular}


Table (5): Distribution of studied sample as regarding to medical management.

\begin{tabular}{|l|c|c|}
\hline \multirow{2}{*}{\multicolumn{1}{|c|}{ Treatment }} & \multicolumn{2}{|c|}{ Patients n = (60) } \\
\cline { 2 - 3 } & No & $\%$ \\
\hline Medical treatment & 5 & 8.33 \\
\hline Interventional therapy & & 71.66 \\
PTA without stenting & 43 & 20.00 \\
PTA with stenting & 12 & \\
\hline
\end{tabular}

Table (6): Distribution of studied sample as regarding to complications that developed after PTA.

\begin{tabular}{|l|c|c|}
\hline \multirow{2}{*}{\multicolumn{1}{|c|}{ Complications after PTA }} & \multicolumn{2}{|c|}{ Patients n = (60) } \\
\cline { 2 - 3 } & No & \% \\
\hline Hematoma & 3 & 5.00 \\
\hline Arterial thrombosis & 5 & 8.33 \\
\hline Arterial occlusion & 4 & 6.66 \\
\hline
\end{tabular}

Table (7): Total score of self care patients' knowledge regarding chronic lower limb ischemia.

\begin{tabular}{|l|c|c|}
\hline \multirow{2}{*}{\multicolumn{1}{|c|}{ Total score of knowledge }} & \multicolumn{2}{c|}{ Patients n= (60) } \\
\cline { 2 - 3 } & No & \% \\
\hline Satisfactory & - & 100 \\
\hline Unsatisfactory & 60 & $467 \pm 0.12$ \\
\hline Mean \pm SD & \multicolumn{2}{|c|}{} \\
\hline
\end{tabular}

Table (8): Mean and stander deviation of the studied sample as regarding to their self care level of knowledge regarding chronic lower limb ischemia.

\begin{tabular}{|l|c|}
\hline \multicolumn{1}{|c|}{ Knowledge items } & Patients $\mathbf{n}=\mathbf{( 6 0 )}$ \\
\cline { 2 - 2 } & Mean \pm SD \\
\hline Anatomy \& physiology $(\mathbf{n}=\mathbf{4})$ & 0.0 \\
\hline About disease $(\mathbf{n}=\mathbf{1 0})$ & $0.167 \pm 0.03$ \\
\hline Improvement of circulation in lower limbs $(\mathbf{n}=\mathbf{8})$ & $0.267 \pm 0.079$ \\
\hline Prevention of weakness of circulation in the lower limbs $(\mathbf{n}=\mathbf{8})$ & $1.4 \pm 0.143$ \\
\hline Foot care $(\mathbf{n}=\mathbf{5})$ & $0.453 \pm 0.259$ \\
\hline Diet $(\mathbf{n}=\mathbf{3})$ & 0.0 \\
\hline
\end{tabular}

$n=$ number of questions.

Table (9): Relation between educational level of the studied sample and their self care level of knowledge regarding chronic lower limb ischemia.

\begin{tabular}{|l|c|c|c|c|}
\hline \multirow{2}{*}{\multicolumn{1}{|c|}{ Level of education }} & \multicolumn{4}{|c|}{ Knowledge } \\
\cline { 2 - 5 } & \multicolumn{2}{|c|}{ Satisfactory } & \multicolumn{2}{c|}{ Unsatisfactory } \\
\cline { 2 - 5 } & No & \%o & \% \\
\hline Illiterate & - & - & 37 & 61.66 \\
\hline Read \& write & - & - & 7 & 11.66 \\
\hline Preparatory & - & - & 4 & 6.66 \\
\hline secondary & - & - & 11 & 18.3 \\
\hline University & - & - & 1 & 1.66 \\
\hline
\end{tabular}


Table (1) : Shows that the highest percent of age of the studied sample was the percent of patients that their age from fifty to sixty and more years old. There was a predominance of male (65\%), most of patients were from rural areas. According to marital status and educational level; the highest percent of patients were married and illiterate.

Table (2) :Illustrates that approximately one third of patients were suffering from cardiac disorders and more than one fifth of patients were suffering from neurological disorders.

Table (3) : Reveals that about two thirds of patients were having family history of chronic lower limb ischemia. Nearly half of patients were smokers and hypertensive. And more than half of patients were diabetics.

Table (4) : Illustrates that only five patients presented by intermittent claudication and most of patients had rest pain. Ulceration was apparent in two fifths of patients and more than half of patients had gangrene in the form of dry and wet gangrene.

Table (5) : Demonstrates that the type of medical management that the patients had underwent. The percent (71.66\%)of patients who underwent Percutanous transluminal angioplasty was higher than the percent of medical treatment.

Table (6) : Illustrates that the highest percent of complications that developed after PTA was regarding to arterial thrombosis then to arterial occlusion and the lowest percent was regarding to wound hematoma.

Table (7) : Shows that all patients had unsatisfactory level of knowledge about self care regarding chronic lower limb ischemia.

Table (8) : Illustrates that the mean score level of knowledge of patients was very poor regarding to the following items: their knowledge about chronic lower limb ischemia, methods of improving circulation in the lower limb, methods of prevention of weakness of circulation in the lower limbs, and caring of their feet.

Table (9) : Illustrates that the highest percent of patients who had unsatisfactory level of knowledge were illiterate while the lowest percent was regarding to who had university education.

\section{Discussion}

The present study showed that; the highest percent of age of the studied sample was the percent of patients that their age from fifty to sixty and more years old and male patients were the predominance. These study findings were in the line with a study conducted at vascular surgery department of Assuit University Hospital by Abdel Salam,(2005) entitled as "endovascular interventional surgery for peripheral arterial occlusive disease of the lower extremities on patients with chronic lower limb ischemia" which revealed that; the age of studied sample was above fifty years old too and male presented a higher incidence than female. This also was supported by (Higgins, 2006 and Mohamed, 2000).

According to Pudner,(2010) who stated that; symptomatic chronic lower limb ischemia is commoner in men, the incidence being twice as high as than women $<60$ in men under 60 years old. And rationalized for this difference as female sex hormones are thought to account for this difference, as symptomatic disease is more common post menopause.

As regard to associated systemic disorders; it was found that patients were suffering from associated systemic disorders in the form of cardiac disorders as hypertension and old myocardial infarction. Another associated systemic disorder that the patients were suffering from it was old cerebrovascular accident in the form of transient ischemic attack. This study finding was supported by Bradbury,(2010) who stated that peripheral arterial occlusive disease rarely presents in isolation and patients will often have accompanying coronary, cerebrovascular, and renal diseases.

As regard to risk factors Ostchega et al,(2007) stated that risk factors modifications is fundamental to the treatment of peripheral arterial occlusive disease and emphasized that management should aggressively address the control of modifiable risk factors including tobacco use, hypertension, and diabetes. The present study revealed that; about two thirds of patients were having family history of chronic lower limb ischemia. Nearly half of patients were smokers and hypertensive. And more than half of patients were diabetics.

These study findings are nearly similar to Ahmed, (2003) who carried out a study at vascular surgery department of Assuit University hospital entitled as " endovascular techniques (balloon dilatation stenting and endografting)" which revealed that smoking was apparent in more than two thirds of the studied sample $(68 \%)$, diabetes in more than half of the sample, and hypertension in less than half of the sample. Multiple authors ( Timaran et al., 2003) have reported that the most frequent associated comorbid condition in their series were smoking in most of the sample, hypertension in less than three quarters of the sample, and diabetes in less than one third of the sample.

According to Fontaine,(2005) who classified chronic ischemia according to clinical presentation into four stages; asymptomatic (stage I), intermittent claudication (stage II), rest pain (stage III), ulceration and/or gangrene (stage IV). With analysis of signs 
and symptoms in the present study; it is observed that; the highest percent that the studied sample presented by it; stage three and four and only small number presented by stage II and no one came to the vascular surgery department by stage one.

According to Ridker,(2007) who supported this study finding by explaining that; the true prevalence of peripheral arterial disease has been difficult to determine because most patients are asymptomatic or the symptoms are atypical and many patients may don't report them, especially in diabetics as presence of neuropathy blunts pain perception and makes sever lesions than in non diabetic patients. When the researcher taked the medical history from the patients; most of patients said that "they went to several physicians for seeking about their condition as orthopedic surgeons, and general surgery physicians, and lastly came to the vascular surgeons or their outpatient clinics by presented late stages (III and IV).

After the diagnosis of chronic lower limb ischemia is established by careful history, appropriate physical examination, and investigations; decision on the management of patients usually comes comfortably into place. As most of our patients presented by late stages (III, IV), so the expected management that would be done for them PTA and bypass surgery; surgical intervention was excluded during selection of the sample. The results of the present study showed that; the highest percent was regarding PTA. Marston,(2006) stated that PTA has been proposed as a safe, alternative to lower extremity arterial bypass graft surgery for life limiting claudication. In recent years, with continuing advances in imaging techniques, angioplasty equipment, and endovascular experts; the use of PTA as a primary TTT for critical limb ischemia has been increasing. The lower morbidity and costs and results comparable to those of bypass surgery also support the increasingly significant role of PTA for CLI.

According to Louis,(2007) who emphasized that; patient teaching is important because the patient has the right to know and to be informed about diagnosis, prognosis of illness, treatment options, risks associated with treatments. And assigned patient teaching to the professional nurse and rationalized the advantages of a well designed comprehensive teaching plan that fit patients' unique learning needs that it reduces health care costs and improve the quality of care. As patient teaching help patients make informed decisions about their health care and to become healthier and more independent.

According to Martin,(2008) who stated that; peripheral arterial disease is a lifelong disease. The more information the patient is provided the better equipped he is to manage this chronic disorder. And stressed on the patient must understand the basic Pathophysiology of chronic lower limb ischemia, contributing risk factors and how best to manage them, prescribed medications, exercise programs, and signs to report to his / her health care providers.

The results of the present study showed that; all patients had unsatisfactory level of knowledge about chronic lower limb ischemia. This could be explained by the fact that, patients didn't receive enough information from health care providers. Some patients were lacking interest, and didn't know any information. While others were eager to know but they didn't find the person who had enough time to provide them with enough information. This in the line with a study conducted at Alexandria university hospital by El-Sebasi,(1984) entitled as "Nursing care program for patients with ischemia of the lower limbs managed conservatively in the main Alexandria university hospital" which revealed that; the majority of patients didn't have enough information about this disease "chronic lower limb ischemia".

As regard to patients' knowledge about diet; the results of the present study showed that; all patients didn't have any information about their diet. Lee and Bokovov, (2009) recommended a healthy diet that promote vascular health. It should include a sufficient number and variety of foods from the fruit, vegetables, dairy, grain, and meat groups each day while staying within individual energy needs. In particular, selection of fruit and vegetables from all subgroups is recommended, including dark green, orange, legume, starchy, and other vegetables, as well citrus, berries, melons, and other fruit. In addition, intake of 1-2 servings of fish per week, Soy protein products (e.g. tofu, miso) can be used in place of animal protein to help lower total cholesterol, LDL cholesterol, and triglycerides as well as increased high density lipoprotein. Dietary sodium should be no more than $29 \mathrm{mg} /$ day and avoiding excessive alcohol or food consumption.

As regard to patients' knowledge about caring by their feet; also the results of the present study showed that; all patients didn't have any information about caring by their feet. Tran \& Anand, (2011) stressed on the importance of foot care for patient with chronic lower limb ischemia which include protecting the extremity from trauma, decreasing vasospasm, preventing and controlling infection, and maximizing arterial perfusion. Carful inspection, cleaning, and lubrication of the both feet are advised to prevent cracking of the skin and infection. 


\section{Conclusion}

All patients had unsatisfactory level of knowledge about self care regarding chronic lower limb ischemia at Assiut university hospital.

\section{Recommendations}

- Integration with other medical departments is needed for the provision of care for those patients as those patients suffered from associated systemic disorders.

- Establishment of continuous health teaching program at vascular surgery department \& its outpatient clinics to provide health teaching using booklet \& illustrated pamphlets for each patient especially those who can't read and write as a high percentage in the present study were illiterates.

\section{References}

1. Abdel salam A. El-sayed, (2005): Endovascular interventional surgery for peripheral arterial occlusive disease of the lower extremity, doctoral thesis, Vascular surgery department, faculty of medicine, Assiut University, Eygpt.172-173p.p.

2. Ahmed K. Abd El-Aziz, (2003): Endovascular techniques (Balloon dilatation stenting and endografting. Master thesis. Vascular surgery department, faculty of medicine, Assiut University, Egypt $\mathrm{p} 77$.

3. Bradbury, A., (2010): Epidemiology and etiology of peripheral arterial disease - 25(supp. 1 1 ): 2-8 p.p.

4. El-Sebai, N. Abdel Monem, (1984): Nursing Care Program for Patients with Ischemia of the Lower Limbs Managed Conservatively in the Main Alexandria University Hospital. Doctoral thesis. Medical Surgical Nursing department, Faculty of Nursing, Alexandria University, Egypt. 98-110 p.p.

5. Fontaine K., (2005): Healing practices: Alternative therapies for nursing (2nd ed. ) upper saddle River, N J Prentice Hall Health.

6. Gey, D. Lesho \& Manngold J., (2008): Management of peripheral arterial disease American family physicion, 69,552-533p.p.

7. Higgins J., (2006): Epidemiology of peripheral arterial disease in women 13(1) $14 \mathrm{P}$.

8. Hirsch A., (2009): Practice guide lines for the management of patients with $h$ peripheral arterial disease Journal of the American College of Cardiology. 113 (11): e 463-54 P.P.

9. Lee T. \& Bokovov J., (2009): Understanding Discharge Instructions after Vascular Surgery: an observational study journal of vascular nursing 23(1): 25-29 P.p.

10. Lewis L. Sharon, (2007): Medical Surgical Nursing: Assessment and management of clinical problems. $1^{\text {st }}$ ed. , ch.(3) Mosby company 901920 P.p.

11. Linsky R., (2010) : Med. heath, contemporary management of peripheral arterial disease, 91(10) : 305-308 P.P.

12. Marston W., (2006): Natural history of limbs with arterial insufficiency and chronic ulceration treated without revascularization. J Vasc Surg 44:108-114.

13. Martin V. Borge, (2008) : Med clin (Bare),peripheral arterial disease in diabetic patients: utility of the toe brachial index 130(16): 611-612.

14. Mohmed G. Genaidi, (2000) : The value of routine duplex study of carotid arteries in patients with peripheral arteriosclerosis. Doctoral thesis. Faculty of medicine, Vascular surgery department, Assiut University Hospital, Egypt. $103 \mathrm{p}$.

15. Ostchega Y., Paulose-Ram R., Dillon C., Hughes J., (2007): Prevalence of peripheral arterial disease and risk factors in persons aged 60 and older: data from the National Health and Nutrition Examination Survey. J Am Geriatr Soc 2007; 55:583-589 P.P.

16. Pudner R., (2010): Nursing the Surgical Patient,ch.(12), Second ed., Elsevier Company, London,465-475 p.p.

17. Ridker P., (2007): Base line characteristics of participants in the Jupiter trial, Am J Cardiol., 100: 165-166 P.

18. Rutherford R., (2010): Vascular Surgery $\left(7^{\text {th }}\right.$ ed., Volume 1: IV) ch. 103 lower extremity arterial disease, Philadelphia: Elsevier.

19. Timaran C., Prault T., Steven S., (2003): Iliac artery stenting versus surgical reconstruction for TASC(trans atlantic inter- society courses) type B., and type C iliac lesion. J., Vasc. Surg. 38(2): $272 \mathrm{P}$.

20. Tran H. and Anand S., (2011): Oral Antiplatelet Therapy in Cerebro Vascular Disease- Coronary Artery Disease, and peripheral artery disease, JAMA 292:1867 P.P. 